\title{
Molecular Characterizations, Virulence Determinants and Antimicrobial Resistance Profiles of Methicillin- Resistant Staphylococcus aureus (MRSA) in the North of Iran
}

\section{Ebrahim Kouhsari}

Ilam University of Medical Sciences

\section{Mojgan Hosseini}

Islamic Azad University Eslamshahr Branch

Alireza Ahmadi

Golestan University of Medical Sciences and Health Services

\section{Parand Khadivar}

Golestan University of Medical Sciences and Health Services

\section{Somayeh Rahimi}

Golestan University of Medical Sciences and Health Services

Abolfazl Amini ( $\square$ amini_ab@msn.com )

Golestan University of Medical Sciences and Health Services https://orcid.org/0000-0002-2495-5368

\section{Research}

Keywords: MRSA, MLST, SCCmec typing, spa typing, agr typing, antibiotic resistance, virulence factors

Posted Date: July 17th, 2020

DOI: https://doi.org/10.21203/rs.3.rs-41456/v1

License: (a) (i) This work is licensed under a Creative Commons Attribution 4.0 International License. Read Full License

Version of Record: A version of this preprint was published at Chemotherapy on December 6th, 2021. See the published version at https://doi.org/10.1159/000520482. 


\section{Abstract}

Background: Emergence and prevalence of Methicillin-Resistant Staphylococcus aureus (MRSA) has become a major universal health concern, limiting therapeutic options.

Methods: In the North side of Iran, during the years 2015 to 2017, a total number of 37 MRSA isolates, including 19 clinical isolates from hospitalized patients and 18 colonizing isolates from health care workers were identified from three hospitals, in Gorgan, North of Iran. Antimicrobial susceptibility test was performed using the disk diffusion method and E-test. The presence of virulence and antibiotic resistance determinants were evaluated by PCR. The genotypic characterization was further analyzed using multi-locus sequence, spa, SCCmec, and agr typing.

Results: The frequency of MRSA among $S$. aureus isolates was 38.14\% (37/97). The most frequent $S$. aureus resistant isolates were found to be obstinate against penicillin (98\%) and gentamicin (82.5\%). Additionally, the lowest resistance rates were found against daptomycin (0\%), vancomycin (2.7\%), and quinupristin-dalfopristin (5.4\%). All MRSA isolates were susceptible to daptomycin with $\mathrm{MIC}_{50} / \mathrm{MIC}_{90}$ of $0.25 / 0.5 \mu \mathrm{g} / \mathrm{ml}$. One isolate belonging to the ST239-SCCmeclll/t037 clone (MIC $\geq 16 \mu \mathrm{g} / \mathrm{ml})$ was resistant to vancomycin. All but one isolate that shares the ST22-SCCmec IV/t790 strain were positive for both $t s s t$ and $p v /$ genes. The most predominant MRSA isolates (27\%) were associated with the ST239-SCCmec III/t037 clone; and followed by ST239-SCCmec III/t924 (16.2\%).

Conclusions: In our study, circulating MRSA strains were genetically diverse with a high prevalence of the ST239SCCmeclll/t037 clone. These findings emphasize the need for future and continuous surveillance studies on MRSA to prevent the dissemination of multidrug resistance and existing MRSA clones in an effective manner.

\section{Background}

Methicillin-resistant Staphylococcus aureus (MRSA) is known to be resistant to various antibiotics and produces many virulence factors, which contribute to high treatment failure [1]. Hence, MRSA is one of the main causes of hospital and community-acquired infections worldwide (HA-MRSA, CA-MRSA) [1-3]. MRSA causes infections ranging from skin and soft tissue to deep-seated and severe life-threatening ones (endocarditis, osteomyelitis, necrotizing pneumonia, meningitis, and toxic shock syndrome) [4-7]. The genetic mobile element of staphylococcal cassette chromosome, $\operatorname{mec}(\mathrm{SCC} m e c)$, is a biomarker that is responsible for resistance of $S$. aureus to methicillin and other beta-lactam antibiotics [8,9]. Additionally, cell surface adhesive components and exotoxins are significant virulence factors of MRSA $[9,10]$. MRSA is frequently spread by direct contact with an infected wound or contaminated hands [11]. Previous studies suggested that health care workers' (HCWs) nasal colonization of HA-MRSA strains may also play a significant role in the pathogenesis and epidemiology of infection in both hospital and community settings $[11,12]$. There is scarce data regarding MRSA genotypes in Iran.

It is ascertained that the combination of molecular and epidemiological methods in surveillance investigations could be promising for controlling the emergence, colonization, and dissemination of predominant genetic lineages and provide epidemiological data for tracing the source of infection for clinical and treatment purposes 
$[2,13]$. The geographical differences in the genotypic characteristic of MRSA have been reported [2]. In Asia, there is significant divergence among countries and regions with respect to prevalence of MRSA; in fact, ST22-SCCmeC IV/t790 and ST239-SCCmec III/t037 clones are predominant among patients in Iran [2, 14], and so is ST239-spa t037 and ST5-spa t002 in China [15]. On the other hand, in many regions in Asia [16, 17], sequence type 239 (ST239) is most prevalent, where, in UK, ST36 and ST30 are the most common types [18].

With this background, we are evaluating the molecular characteristics, antibiotic resistance patterns, and virulence genes profiles of MRSA isolates obtained from two kinds of study populations, namely hospitalized patients and health care workers (HCWs) in Gorgan, North of Iran.

\section{Methods}

\section{Study Design and Sample Collection of S. aureus Isolates}

This cross-sectional study was conducted from January 2, 2016 to October 28, 2018 in three hospitals (total of 920-beds) in Gorgan, North of Iran. Written informed consent was obtained from all the patients or HCWs and the study protocol was approved by the Ethics Committee in Golestan University of Medical Sciences (No. 31078693122419), and was conducted in accordance with the Declaration of Helsinki. The demographic profiles of patients and HCWs were recorded. We identified S. aureus and MRSA in hospitalized patients and HCWs as well (Table 1). Only the first sample of each patient was included in the study. In case of HCWs, samples were collected from both anterior nares. 302 unduplicated clinical samples (blood, urine, wound, sputum, and others) were obtained from in-patients, out of which $S$. aureus and MRSA were identified in 53 (17.5\%), and 19 specimens (6.29\%), respectively. Likewise, 351 unduplicated non-clinical samplings from the anterior nares of HCWs were carried out. All the samples were sent for bacterial culturing and identification, using Gram staining, and standard biochemical tests, such as catalase, tube coagulase, DNase test, and mannitol fermentation [19]. The identification process of all $S$. aureus isolates was confirmed by using genotypic methods for the presence of $n u c A$, and femA genes $[2,20]$. Data on department and period of hospitalization, clinical symptoms, antibiotic usages, and underlying conditions were recorded. 
Table 1

Demographic characteristics of patients and healthcare workers (HCWs) at the three clinical center of Gorgan, Iran.

\begin{tabular}{|c|c|c|c|c|c|}
\hline & \multirow[t]{2}{*}{ Group } & \multicolumn{2}{|l|}{ Patients } & \multicolumn{2}{|c|}{ Healthcare workers } \\
\hline & & $\begin{array}{l}\text { Total number } \\
\text { (N) }\end{array}$ & $\begin{array}{l}\text { S. aureus } \\
\mathrm{n}(\%)\end{array}$ & $\begin{array}{l}\text { Total number } \\
\text { (N) }\end{array}$ & $\begin{array}{l}\text { S. aureus } \\
\mathrm{n}(\%)\end{array}$ \\
\hline Prevalence & & 302 & $\begin{array}{l}53 \\
(17.54)\end{array}$ & 351 & $\begin{array}{l}44 \\
(12.53)\end{array}$ \\
\hline \multicolumn{6}{|l|}{ Characteristics } \\
\hline \multirow[t]{5}{*}{ Age } & $<25$ years & 51 & $10(19.6)$ & 46 & $5(10.86)$ \\
\hline & $\begin{array}{l}25- \\
35 \text { years }\end{array}$ & 59 & $\begin{array}{l}15 \\
(25.42)\end{array}$ & 99 & $\begin{array}{l}11 \\
(11.11)\end{array}$ \\
\hline & $\begin{array}{l}36- \\
45 \text { years }\end{array}$ & 70 & $\begin{array}{l}12 \\
(17.14)\end{array}$ & 105 & $\begin{array}{l}14 \\
(13.33)\end{array}$ \\
\hline & $\begin{array}{l}46- \\
55 \text { years }\end{array}$ & 63 & $7(11.11)$ & 71 & $9(12.67)$ \\
\hline & $\geq 56$ years & 59 & $9(15.25)$ & 30 & $5(16.66)$ \\
\hline \multirow[t]{2}{*}{ Sex } & Female & 139 & $\begin{array}{l}23 \\
(16.54)\end{array}$ & 202 & $\begin{array}{l}27 \\
(13.36)\end{array}$ \\
\hline & Male & 163 & $30(18.4)$ & 149 & $17(11.4)$ \\
\hline \multirow{2}{*}{$\begin{array}{l}\text { Duration of } \\
\text { Hospitalization }\end{array}$} & $\leq 7$ days & 216 & $32(14.8)$ & - & - \\
\hline & $\otimes 7$ days & 86 & $21(24.4)$ & - & - \\
\hline
\end{tabular}

\section{Antimicrobial Susceptibility Test}

Antibiotic susceptibility test (AST) was performed using the disc diffusion method based on CLSI guidelines for antibiotics [21]; nitrofurantoin $(300 \mu \mathrm{g})$, gentamicin $(10 \mu \mathrm{g})$, rifampin $(5 \mu \mathrm{g})$, chloramphenicol $(30 \mu \mathrm{g})$, erythromycin $(15 \mu \mathrm{g})$, clindamycin $(2 \mu \mathrm{g})$, tetracycline $(30 \mu \mathrm{g})$, ciprofloxacin $(5 \mu \mathrm{g})$, penicillin (10units), linezolid $(30 \mu \mathrm{g})$, and quinupristin/dalfopristin(15 $\mathrm{g})(\mathrm{BBL} . \mathrm{BD}, \mathrm{USA})$. According to CLSI 2018 [21], the linezolid resistant isolates tested by disk diffusion method were also rechecked using E-test technique.

The MRSA isolates screening was performed using phenotypic (cefoxitin $(30 \mu \mathrm{g})$ ) discs on Mueller-Hinton agar plates and molecular method (mecA gene) [20]. Moreover, the minimum inhibitory concentration (MIC) of mentioned antibiotics and also vancomycin, and daptomycin (bioMérieux, France) in MRSA and MSSA isolates were determined by E-test. S. aureus ATCC 25923 was used as the control for AST. The clinical MRSA isolates were stored in Tryptic soy broth (TSB; Merck; Germany) containing $20 \%$ glycerol at $-70{ }^{\circ} \mathrm{C}$ for further molecular analysis.

\section{Extraction Of Genomic Dna}


Genomic DNA was extracted from fresh overnight cultures of MRSA isolates using the QIAamp DNA mini kit (Qiagen, Hilden, Germany), according to the manufacturer's instructions. DNA purity, quality, and quantity were measured by absorbance spectrophotometry (Nanodrop-1000; NanoDrop Technologies, Wilmington, DE. USA) and agarose gel electrophoresis. Whole extracted DNA from MRSA isolates was immediately stored at $-20^{\circ} \mathrm{C}$ for further analysis.

\section{Molecular Detection Of Resistance, Adhesion, And Toxin Encoding Genes}

All MRSA isolates were screened for drug resistance genes; ermA, ermB, ermC, InuA, fusB, fusC, mphA, $m p h C$, mupA, cat, aacA-aphD, aadC, aphA ${ }_{3}$, tetK, tetM, tetL, vanA, vanB, and $\operatorname{cfr}$.[15, 21-24] Detection of 17 virulence genes including sea, seb, sec, sed, see, seg, sep, eta, etb, hla, hld, hlg, tsst, pvl, clfA, and clfB in 97 S. aureus isolates was performed as previously described $[3,25,26]$.

\section{Molecular Characterizations}

By SCCmec typing analysis, four types (type I, II, III, and IV) were observed among 37 MRSA isolates. The most predominant type was SCC mec III, while 23 isolates (62.16\%) expressed it, followed by type IV (21.6\%, 8/37), type I $(8.1 \%, 3 / 37)$, and type II $(5.4 \%, 2 / 37)$ respectively. Only one isolate was not typeable. All isolates carrying $\mathrm{pvI}$ gene were found to be associated with SCCmec type IV. Distribution of antibiotic resistance profile, virulence genes profile and SCCmectypes among 37 MRSA strains isolated from in-patients and HCWs samples have been shown in Table 6.

Results of the agrtyping method revealed that $64.86 \%, 24.3 \%$, and $8.1 \%$ of 37 MRSA isolates belonged to agr type I, agr type III and agr type II, respectively. Also, one MRSA isolate was non-typeable. The majority (65.2\%) of $\mathrm{SCC} m e c$ III isolates harbored agrgroup I. Correspondingly, agr group III was present among SCCmec III (77.8\%), and IV (22.2\%) isolates and all of agrll group isolates were found in SCCmec IV.

The spa typing categorized MRSA isolates into 8 spa types. Among them, spa t37 was the most prevalent type $(27 \%, 10 / 37)$, followed by t924 (18.9\%, 7/37), t790 (13.5\%, 5/37), t383 (13.5\%, 5/37), and t030 (10.8\%, 4/37). Each of the remaining spa types was characterized in $\leq 3$ isolates.

According to the MLST method among the 37 MRSA isolates, five different profiles (ST239 in 20 strains, ST22 in 6 strains, ST45 in 5 strains, ST15 in 3 strains, and ST585 in 3 strains) were identified. All 8 isolates harbored pvl gene belonged to ST22-SCCmecIV/t790 (62.5\%), and ST15-SCCmecIV/t084 clones (37.5\%). Of the total 11 isolates harboring tsst encoding gene, 7 (63.63\%) belonged to ST239-SCC mec III/t037 clone and 4 (36.37\%) were member of ST22-SCC mec IV/t790 clone. Table 6 lists the characteristics of the 37 MRSA molecular types, studied. ST239-SCCmecllI/t037, ST22-SCCmeclll/t016, ST22-SCCmeclV/t790, and ST45-SCCmed/t383 clones were most diverse ones in the three clinical centers in Gorgan. Moreover, Fig. 1 depicts the distribution of MRSA types at three clinical centers in Gorgan, Iran.

\section{Results}




\section{Prevalence of Clinical and Non-Clinical S. aureus Isolates}

In a total number of 97 S. aureus isolates, 44/351 (12.53\%) and 53/302 (17.54\%) cases were from HCWs and hospitalized patients, respectively (Table 1). In samples obtained from HCWs, $10.6 \%(14 / 132)$ and $9.91 \%$ $(12 / 121)$ of all $S$. aureus isolates belonged to the nurses and technicians, respectively (Table 2$)$. The clinical $S$. aureus isolates were collected from different sources, including urine 14 (13.3\%); wound 18 (21.95\%); blood 9 (17.3\%); Sputum 5 (18.51\%); Pus 3 (15.8\%), and other body fluids 4 (21\%) (Table 3). Thirty-seven MRSA and sixty methicillin susceptible $S$. aureus (MSSA) strains were recovered from clinical and nasal carriages of HCWs samples, respectively (Tables 2 and 3 ). The proportion of HCWs with MRSA nasal carriage was $48.6 \%(18 / 37)$. Most of the MRSA isolates were obtained from wounds [18.9\% (7 isolates)], and urine [16.2\% (6 isolates)] (Table 3).S. aureus along with MRSA was identified in 44 (12.53\%) and 18 specimens (5.12\%), respectively.

Table 2

Distribution of $S$. aureus, MRSA and MSSA carriage among the different health professions

\begin{tabular}{|lllll|}
\hline HCW & No. of samples & $\begin{array}{l}\text { S. aureus } \\
\mathbf{n}(\%)\end{array}$ & $\begin{array}{l}\text { MRSA } \\
\mathbf{n}(\%)\end{array}$ & $\begin{array}{l}\text { MSSA } \\
\mathbf{n}(\%)\end{array}$ \\
\hline Doctors & 34 & $7(20.5)$ & $2(5.88)$ & $5(14.7)$ \\
\hline Nurses & 132 & $14(10.6)$ & $5(3.78)$ & $9(6.81)$ \\
\hline Technicians & 121 & $12(9.91)$ & $6(4.95)$ & $6(4.95)$ \\
\hline midwives & 12 & $3(25)$ & $1(8.33)$ & $2(16.66)$ \\
\hline Radiologist & 4 & $0(0)$ & $0(0)$ & $0(0)$ \\
\hline Pharmacist & 6 & $1(16.66)$ & $0(0)$ & $1(16.66)$ \\
\hline Others & 42 & $7(16.66)$ & $4(9.52)$ & $3(7.14)$ \\
\hline Total & 351 & $44(12.53)$ & $18(5.12)$ & $26(7.4)$ \\
\hline
\end{tabular}


Table 3

Distribution of $S$. aureus, MRSA and MSSA carriage in clinical samples

\begin{tabular}{|c|c|c|c|c|}
\hline Site of Sample Collection/N. & Ward/N. & $\begin{array}{l}\text { S. aureus } \\
\text { n (\%) }\end{array}$ & $\begin{array}{l}\text { MRSA } \\
\text { n (\%) }\end{array}$ & $\begin{array}{l}\text { MSSA } \\
\text { n (\%) }\end{array}$ \\
\hline \multirow[t]{7}{*}{ Blood/52 } & $\mathrm{ICU} / 3$ & $9(17.3)$ & $3(5.76)$ & $6(11.53)$ \\
\hline & $\mathrm{CCU} / 5$ & & & \\
\hline & Surgery/10 & & & \\
\hline & Emergency/8 & & & \\
\hline & Burn/13 & & & \\
\hline & Internal/9 & & & \\
\hline & ENT/4 & & & \\
\hline \multirow[t]{7}{*}{ Urine/105 } & $\mathrm{ICU} / 0$ & 14 (13.33) & $6(5.71)$ & $8(7.61)$ \\
\hline & $\mathrm{CCU} / 35$ & & & \\
\hline & Surgery/25 & & & \\
\hline & Emergency/21 & & & \\
\hline & Burn/14 & & & \\
\hline & Internal/10 & & & \\
\hline & ENT/0 & & & \\
\hline \multirow[t]{7}{*}{ Wound/82 } & $\mathrm{ICU} / 8$ & 18 (21.95) & $7(8.53)$ & $11(13.41)$ \\
\hline & $\mathrm{CCU} / 9$ & & & \\
\hline & Surgery/17 & & & \\
\hline & Emergency/17 & & & \\
\hline & Burn/8 & & & \\
\hline & Internal/10 & & & \\
\hline & ENT/13 & & & \\
\hline \multirow[t]{7}{*}{ Sputum/27 } & $\mathrm{ICU} / 3$ & $5(18.51)$ & $0(0)$ & $5(18.51)$ \\
\hline & $\mathrm{CCU} / 0$ & & & \\
\hline & Surgery/3 & & & \\
\hline & Emergency/5 & & & \\
\hline & Burn/2 & & & \\
\hline & Internal/6 & & & \\
\hline & ENT/8 & & & \\
\hline
\end{tabular}




\begin{tabular}{|c|c|c|c|c|}
\hline Site of Sample Collection/N. & Ward/N. & $\begin{array}{l}\text { S. aureus } \\
\mathrm{n}(\%)\end{array}$ & $\begin{array}{l}\text { MRSA } \\
\mathrm{n}(\%)\end{array}$ & $\begin{array}{l}\text { MSSA } \\
\mathrm{n}(\%)\end{array}$ \\
\hline \multirow[t]{7}{*}{ Body Fluids/19 } & $\mathrm{ICU} / 0$ & $4(21.05)$ & $2(10.52)$ & $2(10.52)$ \\
\hline & $\mathrm{CCU} / 0$ & & & \\
\hline & Surgery/4 & & & \\
\hline & Emergency/5 & & & \\
\hline & Burn/10 & & & \\
\hline & Internal/0 & & & \\
\hline & ENT/0 & & & \\
\hline \multirow[t]{7}{*}{ Pus/17 } & $\mathrm{ICU} / 2$ & $3(15.79)$ & $1(5.26)$ & $2(10.52)$ \\
\hline & $\mathrm{CCU} / 0$ & & & \\
\hline & Surgery $/ 5$ & & & \\
\hline & Emergency/2 & & & \\
\hline & Burn/7 & & & \\
\hline & Internal/0 & & & \\
\hline & $\mathrm{ENT} / 1$ & & & \\
\hline \multirow[t]{7}{*}{ Total/302 } & $\mathrm{ICU} / 16$ & $53(17.54)$ & 19 (6.29) & $34(11.25)$ \\
\hline & $\mathrm{CCU} / 49$ & & & \\
\hline & Surgery/64 & & & \\
\hline & Emergency/58 & & & \\
\hline & Burn/56 & & & \\
\hline & Internal/35 & & & \\
\hline & ENT/26 & & & \\
\hline
\end{tabular}

\section{Antimicrobial Susceptibility}

S. aureus isolates were found to be predominantly resistant to penicillin $(98 \%, 95 / 97)$ and gentamicin $(82.5 \%$, 80/97). Similarly, $5.4 \%(2 / 37)$ and $18.9 \%$ (7/37) of MRSA isolates were resistant to quinupristin-dalfopristin and linezolid (Table 4). The MIC ranges, MIC50 and $\mathrm{MIC}_{90}$ values for 14 antibiotics in MRSA and MSSA isolates are shown in Table 5. E-test strips results revealed that in entire MRSA isolates only one (2.7\%) was resistant to vancomycin $(\geq 16 \mu \mathrm{g} / \mathrm{ml})$ and $6(16.08 \%)$ were obstinate against linezolid $(\geq 8 \mu \mathrm{g} / \mathrm{ml})$. All the MRSA isolates were susceptible to daptomycin with $\mathrm{MIC}_{50}$ of $0.25 \mu \mathrm{g} / \mathrm{ml}$ and $\mathrm{MIC}_{90}$ of $0.5 \mu \mathrm{g} / \mathrm{ml} .5 .4 \%(2 / 6)$ of the linezolid resistant MRSA isolates showed high resistance with MIC of $64 \mu \mathrm{g} / \mathrm{ml}$ to the respective antibiotic. 
Table 4

Antibiotic resistance of $S$. aureus, MRSA and MSSA isolates among nasal carriage and clinical samples by disk diffusion method

\begin{tabular}{|llll|}
\hline Antimicrobial Agent & S. aureus (n= 97) & MRSA (n= 37) & MSSA (n= 60) \\
\cline { 2 - 4 } & $\mathbf{R}, \mathbf{n}(\%)$ & $\mathbf{R}, \mathbf{n}(\%)$ & $\mathbf{R , ~ n}(\%)$ \\
\hline Penicillin (P) & $95(98)$ & $37(100)$ & $58(96.5)$ \\
\hline Cefoxitin (CF) & $37(38.1)$ & $37(100)$ & $0(0)$ \\
\hline Gentamicin (GM) & $80(82.5)$ & $32(86.5)$ & $48(80)$ \\
\hline Erythromycin (E) & $46(47.4)$ & $34(91.9)$ & $12(20)$ \\
\hline Tetracycline (TE) & $35(36.1)$ & $25(67.5)$ & $10(16.6)$ \\
\hline Ciprofloxacin (CIP) & $33(34)$ & $23(62.1)$ & $10(16.6)$ \\
\hline Nitrofurantoin (FD) & $39(40.2)$ & $30(81.1)$ & $9(15)$ \\
\hline Clindamycin (CC) & $35(36.1)$ & $27(73)$ & $8(13.3)$ \\
\hline Chloramphenicol (C) & $40(41.2)$ & $30(81.1)$ & $10(16.6)$ \\
\hline Rifampin (RIF) & $26(26.8)$ & $21(56.7)$ & $5(8.3)$ \\
\hline Quinupristin-Dalfopristin (SYN) & $2(2.1)$ & $2(5.4)$ & $0(0.0)$ \\
\hline Linezolid (LNZ) & $8(8.2)$ & $7(18.9)$ & $1(1.6)$ \\
\hline
\end{tabular}


Table 5

Minimal Inhibitory Concentrations (MICs) of MRSA and MSSA isolates

\begin{tabular}{|c|c|c|c|c|c|c|c|c|c|c|}
\hline \multirow{2}{*}{$\begin{array}{l}\text { Antimicrobial } \\
\text { Agent }\end{array}$} & \multirow{2}{*}{$\begin{array}{l}\text { MIC } \\
\text { Range } \\
(\mu \mathrm{g} / \mathrm{mL})\end{array}$} & \multirow{2}{*}{$\begin{array}{l}\text { Breakpoints } \\
\text { (CLSI) } \\
(\mu \mathrm{g} / \mathrm{mL}) \\
(\mathrm{S} / \mathrm{I} / \mathrm{R})\end{array}$} & \multicolumn{4}{|c|}{ MRSA (n:37) } & \multicolumn{4}{|c|}{ MSSA (n:60) } \\
\hline & & & $\% S$ & $\% R$ & $\begin{array}{l}\text { MIC } \\
50\end{array}$ & $\begin{array}{l}\text { MIC } \\
90\end{array}$ & $\% \mathrm{~S}$ & $\% \mathrm{R}$ & $\begin{array}{l}\text { MIC } \\
50\end{array}$ & $\begin{array}{l}\text { MIC } \\
90\end{array}$ \\
\hline Penicillin $(P)$ & $\begin{array}{l}0.125- \\
>256\end{array}$ & $\begin{array}{l}\leq 0.12 /-/ \\
\geq 0.25\end{array}$ & 0 & 100 & 64 & 128 & 3.5 & 96.5 & 16 & 256 \\
\hline Cefoxitin (CF) & $\begin{array}{l}0.25- \\
>128\end{array}$ & $\leq 4 /-/ \geq 8$ & 0 & 100 & 64 & 128 & 100 & 0 & 0.256 & 1 \\
\hline $\begin{array}{l}\text { Vancomycin } \\
\text { (VA) }\end{array}$ & $\begin{array}{l}<0.063- \\
16\end{array}$ & $\begin{array}{l}\leq 2 / 4-8 / \\
\geq 16\end{array}$ & 83.8 & 2.7 & 1 & 8 & 100 & 0 & 1 & 1 \\
\hline $\begin{array}{l}\text { Daptomycin } \\
(\mathrm{DAP})\end{array}$ & $0.063-1$ & $\leq 1 /-/$ & 100 & 0 & 0.25 & 0.5 & 100 & 0 & 0.25 & 0.5 \\
\hline $\begin{array}{l}\text { Gentamicin } \\
(\mathrm{GM})\end{array}$ & $\begin{array}{l}0.5- \\
>512\end{array}$ & $\begin{array}{l}\leq 4 / 8 / \\
\geq 16\end{array}$ & 10.9 & 89.1 & 64 & 512 & 20 & 80 & 32 & 256 \\
\hline Erythromycin (E) & $\begin{array}{l}0.25- \\
>128\end{array}$ & $\begin{array}{l}\leq 0.5 / 1-4 \\
/ \geq 8\end{array}$ & 2.1 & 96.5 & 128 & 128 & 81.6 & 18.3 & 1 & 64 \\
\hline Tetracycline (TE) & $\begin{array}{l}0.5- \\
256\end{array}$ & $\begin{array}{l}\leq 4 / 8 / \\
\geq 16\end{array}$ & 25.87 & 68.53 & 32 & 128 & 81.6 & 18.3 & 8 & 32 \\
\hline $\begin{array}{l}\text { Ciprofloxacin } \\
(\mathrm{CIP})\end{array}$ & $\begin{array}{l}0.25- \\
>128\end{array}$ & $\begin{array}{l}\leq 1 / 2 / \\
\geq 4\end{array}$ & 41.26 & 52.45 & 4 & 32 & 81.6 & 18.3 & 2 & 32 \\
\hline $\begin{array}{l}\text { Nitrofurantoin } \\
\text { (FD) }\end{array}$ & $2->512$ & $\begin{array}{l}\leq 32 / 64 / \\
\geq 128\end{array}$ & 21.62 & 78.37 & 128 & 512 & 86.7 & 13.3 & 16 & 64 \\
\hline $\begin{array}{l}\text { Clindamycin } \\
\text { (CC) }\end{array}$ & $\begin{array}{l}0.25- \\
>128\end{array}$ & $\begin{array}{l}\leq 0.5 / 1-2 \\
/ \geq 4\end{array}$ & 32.43 & 67.56 & 4 & 32 & 83.3 & 16.6 & 0.5 & 16 \\
\hline $\begin{array}{l}\text { Chloramphenicol } \\
\text { (C) }\end{array}$ & $8->128$ & $\begin{array}{l}\leq 8 / 16 / \\
\geq 32\end{array}$ & 24.3 & 75.6 & 128 & 128 & 80 & 20 & 8 & 32 \\
\hline Rifampin (RIF) & $\begin{array}{l}<0.063- \\
>128\end{array}$ & $\begin{array}{l}\leq 1 / 2 / \\
\geq 4\end{array}$ & 35.66 & 51.05 & 4 & 64 & 80 & 10 & 2 & 16 \\
\hline $\begin{array}{l}\text { Quinupristin- } \\
\text { Dalfopristin } \\
\text { (SYN) }\end{array}$ & $\begin{array}{l}0.25- \\
>128\end{array}$ & $\begin{array}{l}\leq 1 / 2 / \\
\geq 4\end{array}$ & 94.6 & 5.4 & 0.5 & 3 & 100 & 0 & 0.25 & 1 \\
\hline Linezolid (LNZ) & $\begin{array}{l}<0.063- \\
64\end{array}$ & $\leq 4 /-/ \geq 8$ & 83.92 & 16.08 & 1 & 8 & 100 & 0 & 0.5 & 4 \\
\hline
\end{tabular}

All of the MRSA isolates in the present study were multidrug resistant (MDR). The main MDR profile among the MRSA isolates included a resistance profile to ten antibiotics (40.5\%; 15/37), six antibiotics (13.5\%; 5/37), nine antibiotics (13.5\%; 5/37), eight antibiotics (10.8\%; 4/37), seven antibiotics (8.1\%; 3/37), five antibiotics (8.1\%; $3 / 37)$, four antibiotics (5.4\%; $2 / 37)$, and eleven antibiotics $(2.7 \% ; 1 / 37)$.

\section{Virulence Gene Profiles}


The distribution of 17 putative adhesion and toxin encoding genes diverged in 37 MRSA strains according to MLST, SCCmec, agr, and spa (Table 6). Nine (24.32\%, 9/37) MRSA isolates harbored $\geq 10$ tested virulence genes, among which were 1 isolate with 12 genes, 4 isolates with 11 genes, and 4 isolates with 10 genes. All adhesion and toxin encoding genes except etb gene were identified within several strains and all MRSA isolates exhibited carriage of at least 5 virulence genes. Only one MRSA isolate harbored etb gene. Among 37 MRSA strains, the most common toxin encoding genes detected were $h l a(37 ; 100 \%), h l g(23 ; 62.16 \%)$, seb (21; 56.7\%), hld (20; $54.05 \%), \sec / \operatorname{sep}(51.3 \%)$, and $p v /$ gene (21.6\%), respectively. All of the $p v /$ positive strains expressed resistance profile to at least 5 antibiotics. The majority $(5 ; 62.5 \%)$ of $p v /$ positive strains belonged to only hospital number 1 and age group of 36 to 56 years. Tested adhesion $c / f A$ and $c / f B$ genes were detected in 36 (97.3\%), and 19 (51.35\%) MRSA strains, respectively.

\section{Table 6}

Characteristics of the 37 MRSA strains in hospitalized patients and HCWs samples at the three clinical center in Gorgan, Iran. 


\begin{tabular}{|c|c|c|c|c|c|c|}
\hline $\begin{array}{l}\text { MRSA } \\
\text { clones }\end{array}$ & $\begin{array}{l}\text { agr } \\
\text { type }\end{array}$ & $\begin{array}{l}\text { Adhesion } \\
\text { and Toxin } \\
\text { profile (No; } \\
\%)\end{array}$ & $\begin{array}{l}\text { Antibiotic } \\
\text { resistance } \\
\text { profile } \\
(\text { No; \%) }\end{array}$ & $\begin{array}{l}\text { Resistant genes profile } \\
\text { (No; \%) }\end{array}$ & $\begin{array}{l}\text { Site of } \\
\text { sample } \\
\text { collection }\end{array}$ & $\begin{array}{l}\text { Strains } \\
\text { (No; \%) }\end{array}$ \\
\hline \multirow[t]{8}{*}{$\begin{array}{l}\text { ST239- } \\
\text { SCCmec } \\
\text { III/ t037 }\end{array}$} & \multirow[t]{8}{*}{1} & \multirow{8}{*}{$\begin{array}{l}\text { seb(5;50), } \\
\text { sec( }(3 ; 30), \\
\text { sed(55;50), } \\
\text { see }(; 10), \\
\text { seg( } 6 ; 60), \\
\text { sep( }(7 ; 70), \\
\text { hla( }(10 ; 10), \\
\text { hld(5;50), } \\
\text { hlg(5;50), } \\
\text { tsst(t7;0), } \\
\text { clfa(10;10), } \\
\text { clfb }(6 ; 60)\end{array}$} & $\begin{array}{l}\mathrm{P}, \mathrm{CF}, \mathrm{GM}, \\
\mathrm{E}, \mathrm{TE}, \mathrm{CIP}, \\
\text { CC, C, RIF, } \\
\text { LNZ } \\
(2 ; 20)\end{array}$ & \multirow[t]{8}{*}{$\begin{array}{l}\operatorname{aacA}-\operatorname{aphD}(7 ; 70), \operatorname{aadC}(1 ; 10), \\
\operatorname{aph} A_{3}(1 ; 10), \operatorname{erm} A(5 ; 50), \operatorname{erm} C(4 ; 40), \\
\operatorname{Inu}(4 ; 40), \operatorname{tet} M(4 ; 40), \operatorname{tet} K(2 ; 20), \\
\operatorname{cat}(8 ; 80), \operatorname{cfr}(2 ; 33.3), \\
\operatorname{van} A, \operatorname{van} B(1 ; 10)\end{array}$} & \multirow[t]{8}{*}{$\begin{array}{l}\text { Nasal } \\
\text { Swab, } \\
\text { Wound, } \\
\text { Blood, } \\
\text { and Urine }\end{array}$} & \multirow[t]{8}{*}{$10 ; 27$} \\
\hline & & & $\begin{array}{l}\text { P, CF, GM, } \\
\text { E, TE, FD, } \\
\text { CC, C, RIF } \\
(2 ; 20)\end{array}$ & & & \\
\hline & & & $\begin{array}{l}\text { P, CF, VA, } \\
\text { GM, E, FD, } \\
\text { CC, C, RIF } \\
(1 ; 10)\end{array}$ & & & \\
\hline & & & $\begin{array}{l}\text { P, CF, GM, } \\
\text { E, CIP, FD, } \\
\text { CC, C, RIF, } \\
\text { LNZ } \\
(1 ; 10)\end{array}$ & & & \\
\hline & & & $\begin{array}{l}P_{,} C F_{,} E_{1} \\
F D(1 ; 10)\end{array}$ & & & \\
\hline & & & $\begin{array}{l}\text { P, CF, GM, } \\
\text { E, TE, CIP, } \\
\text { FD, C } \\
(1 ; 10)\end{array}$ & & & \\
\hline & & & $\begin{array}{l}\text { P, CF, CIP, } \\
\text { FD, CC } \\
(1 ; 10)\end{array}$ & & & \\
\hline & & & $\begin{array}{l}\text { P, CF, GM, } \\
\text { E, TE, CIP, } \\
\text { FD, C, RIF } \\
(1 ; 10)\end{array}$ & & & \\
\hline \multirow{5}{*}{$\begin{array}{l}\text { ST239- } \\
\text { SCCmec } \\
\text { III/ t924 }\end{array}$} & \multirow[t]{5}{*}{ III } & \multirow{5}{*}{$\begin{array}{l}\text { sea(1;16.6), } \\
\text { seb }(4 ; 66.6), \\
\text { sec }(4 ; 66.6), \\
\text { sed }(1 ; 16.6), \\
\text { see }(1 ; 16.6), \\
\text { seg }(1 ; 16.6), \\
\text { sep }(2 ; 33.3), \\
\text { eta(1;16.6), } \\
\text { hla }(6 ; 100), \\
\text { hld }(4 ; 66.6), \\
\text { hlg(5;83.3), } \\
\text { clfa(6;100), } \\
\text { clfb }(3 ; 50)\end{array}$} & $\begin{array}{l}\mathrm{P}, \mathrm{CF}, \mathrm{GM}, \\
\mathrm{E}, \mathrm{TE}, \mathrm{CIP}\end{array}$ & \multirow{5}{*}{$\begin{array}{l}\text { aacA-aphD(4;66.6), aadC(1;16.6), } \\
\operatorname{aph} A_{3}(1 ; 16.6), \operatorname{erm} A(3 ; 50), \\
\operatorname{erm} B(1 ; 16.6), \operatorname{erm} C(1 ; 16.6), \\
\operatorname{lnu} A(2 ; 33.3), \operatorname{tetK}(2 ; 33.3), \\
\text { fusc }(1 ; 16.6), \operatorname{cat}(5 ; 83.3), \operatorname{cfr}(2 ; 33.3)\end{array}$} & \multirow{5}{*}{$\begin{array}{l}\text { Nasal } \\
\text { Swab, } \\
\text { Urine, } \\
\text { Body } \\
\text { Fluids, } \\
\text { and Pus }\end{array}$} & \multirow[t]{5}{*}{$6 ; 16.2$} \\
\hline & & & $\begin{array}{l}\text { FD, CC, C, } \\
\text { LNZ } \\
(1 ; 16.6)\end{array}$ & & & \\
\hline & & & $\begin{array}{l}\text { P, CF, GM, } \\
\text { E, FD, C } \\
(1 ; 16.6)\end{array}$ & & & \\
\hline & & & $\begin{array}{l}\text { P, CF, GM, } \\
\text { CIP, FD, } \\
\text { CC } \\
(1 ; 16.6)\end{array}$ & & & \\
\hline & & & $\begin{array}{l}\text { P, CF, GM, } \\
\text { E,TE, CIP, } \\
\text { FD, CC, C, } \\
\text { RIF } \\
(1 ; 16.6)\end{array}$ & & & \\
\hline
\end{tabular}




\begin{tabular}{|c|c|c|c|c|c|c|}
\hline & & & $\begin{array}{l}\text { P, CF, GM, } \\
\text { E, TE, CIP, } \\
\text { CC, C, RIF, } \\
\text { LNZ } \\
(1 ; 16.6) \\
\text { P, CF, GM, } \\
\text { E, FD, CC, } \\
\text { C, RIF } \\
(1 ; 16.6)\end{array}$ & & & \\
\hline $\begin{array}{l}\text { ST22- } \\
\text { SCCmec } \\
\text { IV/t790 }\end{array}$ & 1 & $\begin{array}{l}\text { sea(2;66.6), } \\
\text { seb( }(3 ; 100), \\
\sec (3 ; 100), \\
\text { sep(3;100), } \\
\text { hla(3;100), } \\
\text { hld(2;66.6), } \\
\text { hlg( } 2 ; 66.6), \\
\text { tsst( } 3 ; 100), \\
\text { pvl( } 3 ; 100), \\
\text { clfa( }(3 ; 100), \\
\text { clfb }(1 ; 33.3)\end{array}$ & $\begin{array}{l}\text { P, CF, GM, } \\
\text { E, TE, FD, } \\
\text { C }(1 ; 33.3) \\
\text { P, CF, GM, } \\
\text { E, TE, CIP, } \\
\text { CC, C, RIF } \\
(1 ; 33.3) \\
\text { P, CF, GM, } \\
\text { E, TE, CIP, } \\
\text { FD, CC, C, } \\
\text { RIF, LNZ } \\
(1 ; 33.3)\end{array}$ & $\begin{array}{l}\text { aacA-aphD(1;33.3), ermA(1;33.3), } \\
\operatorname{ermC}(2 ; 66.6), \operatorname{tetK}(2 ; 66.6), \\
\operatorname{tetM}(1 ; 33.3), \\
\operatorname{cat}(3 ; 100), \ln u A(2 ; 66.6), \operatorname{aph} A_{3}(1 ; 33.3) \text {, } \\
\text { fusc }(1 ; 33.3), \operatorname{cfr}(1 ; 16.6)\end{array}$ & $\begin{array}{l}\text { Nasal } \\
\text { Swab }\end{array}$ & $3 ; 8.1$ \\
\hline $\begin{array}{l}\text { ST239- } \\
\text { SCCmec } \\
\text { III/t030 }\end{array}$ & 1 & $\begin{array}{l}\text { seb(3;100), } \\
\text { eta(3;100), } \\
\text { hla(3;100), } \\
\text { hld(2;66.6), } \\
\text { hlg(3;100), } \\
\text { clfa(3;100), } \\
\text { clfb }(1 ; 33.3)\end{array}$ & $\begin{array}{l}\text { P, CF, GM, } \\
\text { E, TE, CIP, } \\
\text { FD, CC, C } \\
(1 ; 33.3) \\
\text { P, CF, GM, } \\
\text { E, TE, FD, } \\
\text { C, RIF } \\
(1 ; 33.3) \\
\text { P, CF, E, } \\
\text { TE, CIP, } \\
\text { FD, CC } \\
(1 ; 33.3)\end{array}$ & $\begin{array}{l}\text { aacA-aphD(1;33.3), ermC(3;100), } \\
\ln u A(1 ; 33.3), \operatorname{tetM}(2 ; 66.6), \operatorname{cat}(1 ; 33.3)\end{array}$ & $\begin{array}{l}\text { Nasal } \\
\text { Swab }\end{array}$ & $3 ; 8.1$ \\
\hline $\begin{array}{l}\text { ST15- } \\
\text { SCCmec } \\
\text { IV/ t084 }\end{array}$ & II & $\begin{array}{l}\operatorname{seb}(2 ; 66.6), \\
\sec (1 ; 33.3), \\
\operatorname{see}(1 ; 33.3), \\
\operatorname{sed}(1 ; 33.3), \\
\operatorname{seg}(2 ; 66.6), \\
\operatorname{sep}(2 ; 66.6), \\
\text { hla( }(3 ; 100), \\
\text { hld }(1 ; 33.3), \\
\text { hlg(1;33.3), } \\
\text { pvl( }(3 ; 100), \\
\text { clfa( }(3 ; 100), \\
\text { clfb }(1 ; 33.3)\end{array}$ & $\begin{array}{l}\text { P, CF, GM, } \\
\text { E, TE, CIP, } \\
\text { FD, CC, C, } \\
\text { RIF } \\
(2 ; 66.6) \\
\\
\text { P, CF, GM, } \\
\text { E, TE, FD, } \\
\text { C }(1 ; 33.3)\end{array}$ & $\begin{array}{l}\text { aacA-aphD(3;100), ermA(2;66.6), } \\
\text { ermC(1;33.3), InuA(2;66.6), } \\
\operatorname{tet} K(1 ; 33.3), \operatorname{tetM}(2 ; 66.6), \operatorname{cat}(3 ; 100)\end{array}$ & $\begin{array}{l}\text { Nasal } \\
\text { Swab, } \\
\text { Wound, } \\
\text { and } \\
\text { Blood }\end{array}$ & $3 ; 8.1$ \\
\hline $\begin{array}{l}\text { ST } 45- \\
\text { SCCmec } \\
\text { I/ t383 }\end{array}$ & 1 & $\begin{array}{l}\text { sea(1;33.3), } \\
\operatorname{seb}(2 ; 66.6), \\
\sec (2 ; 66.6), \\
\operatorname{sep}(2 ; 66.6), \\
\text { eta(1;33.3), } \\
\text { hla }(3 ; 100), \\
\text { hld }(1 ; 33.3), \\
\text { hlg }(2 ; 66.6), \\
\text { clfa( }(3 ; 100), \\
\text { clfb }(1 ; 33.3)\end{array}$ & $\begin{array}{l}\text { P, CF, GM, } \\
\text { E, TE, CIP, } \\
\text { FD, CC, C, } \\
\text { RIF } \\
(2 ; 66.6) \\
\text { P, CF, GM, } \\
\text { E, TE, FD } \\
(1 ; 33.3)\end{array}$ & $\begin{array}{l}\operatorname{aacA}-a p h D(3 ; 100), \operatorname{erm} C(3 ; 100) \\
\operatorname{tet} K(1 ; 33.3), \operatorname{Inu} A(2 ; 66.6), \operatorname{tet}(1 ; 33.3), \\
\operatorname{tet} M(1 ; 33.3), \operatorname{cat}(2 ; 66.6)\end{array}$ & $\begin{array}{l}\text { Nasal } \\
\text { Swab, } \\
\text { and } \\
\text { Blood }\end{array}$ & $3 ; 8.1$ \\
\hline
\end{tabular}




\begin{tabular}{|c|c|c|c|c|c|c|}
\hline $\begin{array}{l}\text { ST45- } \\
\text { SCCmec } \\
\text { II/ t383 }\end{array}$ & I & $\begin{array}{l}\operatorname{seb}(2 ; 100), \\
\sec (1 ; 50), \\
\operatorname{sep}(2 ; 100), \\
\text { hla }(2 ; 100), \\
\text { hld }(2 ; 100), \\
\text { llg }(; 100), \\
\text { llfa } 2 ; 100), \\
\text { clfb }(1 ; 50)\end{array}$ & $\begin{array}{l}\text { P, CF, GM, } \\
\text { E, TE, CIP, } \\
\text { FD, CC, C, } \\
\text { RIF } \\
(1 ; 100) \\
\text { P, CF, E, } \\
\text { FD, CC, C } \\
(1 ; 100)\end{array}$ & $\begin{array}{l}\text { aacA-aphD(1;50), ermA(1;50), } \\
\text { ermC }(1 ; 50), \ln u A(2 ; 100), \text { tetK }(1 ; 50) \text {, } \\
\text { cat }(2 ; 100)\end{array}$ & $\begin{array}{l}\text { Wound, } \\
\text { and Body } \\
\text { Fluids }\end{array}$ & $2 ; 5.4$ \\
\hline $\begin{array}{l}\text { ST22- } \\
\text { SCCmec } \\
\text { IV/t790 }\end{array}$ & III & $\begin{array}{l}\text { sea }(1 ; 50), \\
\operatorname{seb}(1 ; 50), \\
\sec (2 ; 100), \\
\operatorname{seg}(1 ; 50), \\
\operatorname{sep}(2 ; 100), \\
\text { eta }(1 ; 50), \\
\text { hla }(2 ; 100), \\
\text { tsstt }(1 ; 50), \\
\text { pvll(2;100), } \\
\text { clfa }(2 ; 100)\end{array}$ & $\begin{array}{l}\text { P, CF, GM, } \\
\text { E, TE, CIP, } \\
\text { CC, C, RIF } \\
(1 ; 50) \\
\text { P, CF, GM, } \\
\text { E, FD, C } \\
(1 ; 50)\end{array}$ & $\begin{array}{l}\operatorname{aacA}-\operatorname{aph} D(1 ; 50), \operatorname{aad} C(1 ; 50), \\
\operatorname{erm} A(1 ; 50), \operatorname{erm} C(1 ; 50), \operatorname{tet}(1 ; 50) \text {, } \\
\operatorname{cat}(2 ; 100)\end{array}$ & $\begin{array}{l}\text { Wound, } \\
\text { and Urine }\end{array}$ & $2 ; 5.4$ \\
\hline $\begin{array}{l}\text { ST22- } \\
\text { SCCmec } \\
\text { III/t016 }\end{array}$ & 1 & $\begin{array}{l}\text { seb }(1 ; 100), \\
\text { sec }(1 ; 100), \\
\text { sed }(1 ; 100), \\
\text { sep }(1 ; 100), \\
\text { hla }(1 ; 100), \\
\text { hld }(1 ; 100), \\
\text { hlg }(1 ; 100), \\
\text { clfa }(1 ; 100), \\
\text { clfb }(1 ; 100)\end{array}$ & $\begin{array}{l}\text { P, CF, GM, } \\
\text { E, TE, CIP, } \\
\text { CC, C, RIF } \\
(1 ; 100)\end{array}$ & $\begin{array}{l}\operatorname{aacA-aphD}(1 ; 100), \operatorname{aad} C(1 ; 100), \\
\operatorname{erm} A(1 ; 100), \operatorname{lnu} A(1 ; 100) \\
\operatorname{tet}(1 ; 100), \operatorname{cat}(1 ; 100)\end{array}$ & $\begin{array}{l}\text { Nasal } \\
\text { Swab }\end{array}$ & $1 ; 2.7$ \\
\hline $\begin{array}{l}\text { ST239- } \\
\text { SCCmec } \\
\text { III/t030 }\end{array}$ & NT & $\begin{array}{l}\text { seb }(1 ; 100), \\
\text { sed }(1 ; 100), \\
\text { seg }(1 ; 100), \\
\text { sep }(1 ; 100), \\
\text { eta }(1 ; 100), \\
\text { etb }(1 ; 100), \\
\text { hla }(1 ; 100), \\
\text { hld }(1 ; 100), \\
\text { hlg }(1 ; 100), \\
\text { clfa }(1 ; 100), \\
\text { clfb }(1 ; 100)\end{array}$ & $\begin{array}{l}\text { P, CF, GM, } \\
\text { E, TE, CIP, } \\
\text { CC, C, RIF, } \\
\text { LNZ } \\
(1 ; 100)\end{array}$ & $\begin{array}{l}\text { aacA-aphD(1;100), ermA(1;100), } \\
\text { InuA }(1 ; 100), \text { tetK }(1 ; 100), \operatorname{cat}(1 ; 100) \text {, } \\
\operatorname{cfr}(1 ; 16.6)\end{array}$ & $\begin{array}{l}\text { Nasal } \\
\text { Swab }\end{array}$ & $1 ; 2.7$ \\
\hline $\begin{array}{l}\text { ST585- } \\
\text { SCCmec } \\
\text { NT/ } \\
\text { t713 }\end{array}$ & 1 & $\begin{array}{l}\text { seb(1;100), } \\
\text { sec(1;100), } \\
\text { sep }(1 ; 100), \\
\text { hla }(1 ; 100), \\
\text { clfa( }(1 ; 100)\end{array}$ & $\begin{array}{l}\text { P, CF, GM, } \\
\text { E, FD, C } \\
(1 ; 100)\end{array}$ & $\begin{array}{l}\text { aacA-aphD(1;100), ermC(1;100), } \\
\operatorname{cat}(1 ; 100)\end{array}$ & $\begin{array}{l}\text { Nasal } \\
\text { Swab }\end{array}$ & $1 ; 2.7$ \\
\hline $\begin{array}{l}\text { ST585- } \\
\text { SCCmec } \\
\text { III/ t924 }\end{array}$ & III & $\begin{array}{l}\text { see }(1 ; 100), \\
\text { hla }(1 ; 100), \\
\text { clfa }(1 ; 100), \\
\text { clfb }(1 ; 100)\end{array}$ & $\begin{array}{l}\text { P, CF, GM, } \\
\text { E, FD } \\
(1 ; 100)\end{array}$ & aacA-aphD(1;100), ermC(1;100) & $\begin{array}{l}\text { Nasal } \\
\text { Swab }\end{array}$ & $1 ; 2.7$ \\
\hline $\begin{array}{l}\text { ST585- } \\
\text { SCCmec } \\
\text { III/ t713 }\end{array}$ & 1 & $\begin{array}{l}\text { sec(1;100), } \\
\text { hla }(1 ; 100), \\
\text { clfa(1;100), } \\
\text { clfb }(1 ; 100)\end{array}$ & $\begin{array}{l}\text { P, CF, CIP, } \\
\text { FD } \\
(1 ; 100)\end{array}$ & - & Urine & $1 ; 2.7$ \\
\hline
\end{tabular}


Abbreviations: MRSA, methicillin-resistant Staphylococcus aureus, agr, accessory gene regulator; HCW, Health Care Worker; P, Penicillin; CF, Cefoxitin; VA, Vancomycin; GM, Gentamicin; E, Erythromycin; TE, Tetracycline; CIP, Ciprofloxacin; FD, Nitrofurantoin; CC, Clindamycin; C, Chloramphenicol; RIF, Rifampin; LNZ, Linezolid.

Note: Sea sep, gene encoding staphylococcal enterotoxins; eta and etb, gene encoding exfoliatin; $h l a \sim h l g$, gene encodinga-hemolysin $\gamma$-hemolysin; tsst, gene encoding toxic shock syndrome toxin $1 ; p v l$, gene encoding PantonValentine leukocidin; $c / f A \sim c / f B$, gene adhesion factor.

\section{Molecular Characterizations}

By SCCmec typing analysis, four types (type I, II, III, and IV) were observed among 37 MRSA isolates. The most predominant type was SCCmec III, while 23 isolates (62.16\%) expressed it, followed by type IV (21.6\%, 8/37), type I $(8.1 \%, 3 / 37)$, and type II $(5.4 \%, 2 / 37)$ respectively. Only one isolate was not typeable. All isolates carrying $\mathrm{pvI}$ gene were found to be associated with SCCmec type IV. Distribution of antibiotic resistance profile, virulence genes profile and SCCmectypes among 37 MRSA strains isolated from in-patients and HCWs samples have been shown in Table 6.

Results of the agrtyping method revealed that $64.86 \%, 24.3 \%$, and $8.1 \%$ of 37 MRSA isolates belonged to agr type I, agr type III and agr type II, respectively. Also, one MRSA isolate was non-typeable. The majority (65.2\%) of $\mathrm{SCC} m e c$ III isolates harbored agrgroup I. Correspondingly, agr group III was present among SCCmec III (77.8\%), and IV (22.2\%) isolates and all of agrll group isolates were found in SCCmec IV.

The spa typing categorized MRSA isolates into 8 spa types. Among them, spa $\mathrm{t} 37$ was the most prevalent type $(27 \%, 10 / 37)$, followed by t924 (18.9\%, 7/37), t790 (13.5\%, 5/37), t383 (13.5\%, 5/37), and t030 (10.8\%, 4/37). Each of the remaining spa types was characterized in $\leq 3$ isolates.

According to the MLST method among the 37 MRSA isolates, five different profiles (ST239 in 20 strains, ST22 in 6 strains, ST45 in 5 strains, ST15 in 3 strains, and ST 585 in 3 strains) were identified. All 8 isolates harbored pvl gene belonged to ST22-SCCmecIV/t790 (62.5\%), and ST15-SCCmedV/t084 clones (37.5\%). Of the total 11 isolates harboring tsst encoding gene, 7 (63.63\%) belonged to ST239-SCCmec III/t037 clone and 4 (36.37\%) were member of ST22-SCCmec IV/t790 clone. Table 6 lists the characteristics of the 37 MRSA molecular types, studied. ST239-SCCmeclll/t037, ST22-SCCmeclll/t016, ST22-SCCmeclV/t790, and ST45-SCCmed/t383 clones were most diverse ones in the three clinical centers in Gorgan. Moreover, Figure 1 depicts the distribution of MRSA types at three clinical centers in Gorgan, Iran.

\section{Discussion}

S. aureus is one of the most frequent bacterial pathogens in Iran, causing a variety of infections. Genotypic background and antibiotic susceptibility of MRSA strains vary in terms of geographical locations dynamically [2].

In this study, the genotypic characterization, virulence determinants patterns and antimicrobial resistance profile of 37 MRSA strains isolated from three hospitals in Gorgan, Northern Iran were analyzed. HA-MRSA associated with high morbidity and mortality has developed worldwide, specifically in Iran [31, 32]. A dramatic emergence and expansion of MRSA in different regions of Iran (20.4\%-93.3\%) leads to an increase in the costs of antibiotic therapy and reduction of treatment choices [2, 33, 34]. 
The frequency of MRSA strains diverges in various geographic areas. The relative prevalence (38.14\%) of HAMRSA strains in our study was comparable to the result obtained by Darban et al. (35\%) [35]. However, this prevalence was lower than that reported in America, Europe, Africa, and Asia [26, 36-41]. The reasons for this discrepancy in the prevalence of MRSA may be related to the dissimilar antibiotic usage patterns, contrary infection control policies, sources of the isolates, and the characteristics of the subjects (HCWs and patients). Results from the present study showed that the frequency of MRSA was similar in hospitalized patients (51.4\%) and HCWs (48.6\%). In Iran, the indiscriminate consumption of beta-lactam antibiotics contributes to the spread of resistance of MRSA to these antibiotics [2].

MRSA can arise from MSSA upon specific site integration of SCC mec into the orf X locus in the chromosome of a susceptible isolate [42].

In the present study, prevalence of MSSA was 61.8\% (92.6\% among the various health professions and $88.7 \%$ in clinical samples), which is in line to the study in Gorgan [43], but was lower compared to Khosravi et al. [44], Heidari et al. [45], and Sepehriseresht et al. [46] surveys.

The gentamycin resistance rate in the 37 MRSA strains was found to be $86.5 \%$, which is higher than rates $(60 \%$, $77 \%, 78.2 \%$, and $40.5 \%$ ) reported in other similar studies $[2,8,38]$.

However, low gentamycin resistance rates in Chinese and Iranian MRSA isolates have been reported $[3,15,47]$. All of the MRSA isolates in the present study were MDR which is in relative line with previous reports from Iran[2, 14] and Taiwan [38]. Our findings also suggested that vancomycin and linezolid are potent and effective treatment options for MRSA [35, 40, 41].

In our study, based on MIC results, 8.3\% (5/60) of MSSA strains were found to express intermediate resistance to vancomycin (VISA). However, of these MSSA strains, $3(60 \%)$ had $\mathrm{MIC}=3 \mathrm{mg} / \mathrm{L}$ and the rest $(40 \%)$ had $\mathrm{MIC}=$ $4 \mathrm{mg} / \mathrm{L}$. Although, Hasani et al. study[48] in the Northwest of Iran reported 19 (23.4\%) of MSSA isolates were VISA. Besides, of 60 MSSA isolates, 20 (33.3\%) were MDR, and our findings also proposed that vancomycin, quinupristin-dalfopristin, daptomycin, and linezolid are potent and effective against MSSA isolates.

The diverse genotypic characteristics of HA-MRSA in distinct geographic regions have been established [2]. Consistent to previously published data [49], MRSA SCCmec type III has been found the most prevalent isolate in our study.

Inconsistent with the previous reports, our research in Iran $[50,51]$ revealed that SCCmec type III was the major SCCmec type among MRSA strains in the present study. Similar to Parhizgari et al. [51] and Zetola's et al. [52] The high frequency of MDR-MRSA in our study belonged to SCCmec type III.

Historically, the expression level of the most virulence factors of $S$. aureus is regulated by agr locus [3]. In agreement with our previous reports [14,53], the most common MRSA isolates belonged to agr I (64.86\%), followed by agrtype III (24.3\%). According to the previous data, there is a substantial association between agr type and certain bacterial virulence determinants [54]. In addition to that, various agr types disseminate from one geographic area to another. Similar to our results, the frequency of bacterial virulence determinants including toxin and adhesive genes in MRSA isolates with agrtype I was higher than type III [2]. The agr type I could have a crucial role in the control of staphylococcal virulence determinants. Nevertheless, in contrast with our findings, 
Nowrouzian et al. [54] showed high frequency of toxin coding genes in MRSA isolates belonged to harboring agr type III.

The prevalence of spa types differs with geographic regions, type of samples, and the time of sampling [55]. The most predominant spa types were t032, t008, and t002 in Europe plus t037 and t002 in Asia. In Iran, most MRSA isolates were associated with spa types $\mathrm{t} 701, \mathrm{t} 12311, \mathrm{t} 021, \mathrm{t} 037$, and $\mathrm{t} 790$. Our study showed that t037 was the most prevalent spa type. However, t030 has been reported as the major spa type in Iran, other than t037 [56], suggesting that t037 has been replaced by t030 spa type in the hospitals in Iran. This finding has been similarly reported in China in the year 1994 to $2008[57,58]$.

Sequence type 239 (ST239) in SCCmec III is found to be the most predominant mobile genetic element in Iran. ST239-SCCmec III is characterized into three clades: South American, European, and Asian [2]. The ST239SCCmeclll/t037 clone, which is the oldest pandemic MRSA strain is a major HA-MRSA clone predominated in Iranian hospitals [59]. It seems that this clone could have been transferred from neighboring countries. This finding suggests that the frequency of ST239 clone may be closely related to MRSA infections. In our study, the MRSA strains which are PVL positive belonged to ST22 and ST15, moreover, MDR-MRSA was detected among the STs and this result is partially consistent with a study conducted in UK [32] disclosing the same matter that MDR-MRSA was found in STs, however, PVL positive MRSA strains belonged to ST772, ST5, ST8, ST22, ST59, and ST8t0 in that paper. Nonetheless, in contrast to the current results, Havaei et al. [60] did not identify any MRSA in ST22 strains. In our research, $62.5 \%$ and $37.5 \%$ of MRSA strains carrying the $p v /$ gene belonged to ST22SCC mec IV/t790 and ST15-MRSA IV/t084 clones, respectively. Additionally, all but one isolate that shares the ST22-SCCmec IV/t790 strains were positive for both tsst and pv/genes. This finding is relatively in agreement with recent study conducted in Iran [2] declaring all but one of the ST22-SCCmec IV/t790 strain, harbored the tsst and $p v /$ genes. The antimicrobial resistance profiles frequently differ in ST clones of MRSA [57]. In accordance with results of a study in UK by Ellington et al. [32], in the current study, isolates with ST22-SCCmec IV/t790 were drastically resistant to multiple antibiotic groups.

There was a powerful correlation observed between ST and spa types: ST239 type was principally related to t037 $(50 \%, 10 / 20)$, ST 22 type to $7790(83.3 \%, 5 / 6)$, ST45 type to $\mathrm{t} 383(100 \%, 5 / 5)$, ST15 type to t084 $(100 \%, 3 / 3)$ and ST585 type to $t 713(66.6 \%, 2 / 3)$, respectively. ST239-t037 $(10 / 37,27 \%)$ was observed to be the most common type in current study, followed by ST239-t924 $(6 / 37,16.2 \%)$. There is a diversity of HA-MRSA clones worldwide, for example, the most predominant clones have developed in many different regions of Asia $[2,59,61-63]$ are ST6-SCCmec IV, ST239-SCCmec III, ST239-SCCmec IV, ST239-SCCmec II, ST5- SCCmec II, and ST30-SCCmec IV. Especially, six genetic types (ST22, ST859, ST291, ST239, ST6, and ST30) are the main clones dominating in Tehran. In this study, $27.01 \%$ of the MRSA isolates belonging to the ST239-SCCmec III/t037 clone were detected as the main clone followed by ST239-SCCmec III/t924 (16.2\%) and ST22-SCCmec IV/t790 (8.1\%) and that have been reported in other previous studies in Iran $[2,14]$. The most VRSA strains separated from clinical samples in USA belonged to ST5 [2].

In line with Shekarabi et al.'s study in 2017 [64], in the current study, one vancomycin resistant (MIC $\geq 16 \mu \mathrm{g} / \mathrm{ml}$ ) isolate was obtained from a 45-year-old woman that showed SCCmec type III, spa type t037, and ST239 as its genetic characteristics. In the recently published data from Iran Azimian et al. [20] and Goudarzi et al. [2] reported the ST1283-SCCmec III/t037 and ST22-SCCmec IV/t790 clones as VRSA isolates, respectively. On other hand, VRSA strains have certain discrepancies in genetic characteristics [65]. The emergence of reduced or full -

Page $17 / 25$ 
resistance to vancomycin in bacterial strains in Iran may be attributed to the selective pressure caused by unsuitable consumption of drugs as the main treatment option available for MRSA-infections [31, 38].

\section{Conclusions}

There is a simultaneous carriage of virulence determinants, multidrug-resistance genes and high genetic diversity among the MRSA strains isolated from patients and HCWs in North of Iran. Sequence typing analysis showed that ST239-SCC mec III/t037and ST22-SCCmec IV/t790 clones have a high expression level of tsst and pv/genes with multidrug resistance genes. Hospital infection control policies and nationwide surveillance efforts are highly demanded to monitor the clonal expansion of MRSA species in the North of Iran.

\section{Abbreviations}

S. aureus

Staphylococcus aureus

MRSA

methicillin-resistant Staphylococcus aureus

MSSA

methicillin-susceptible Staphylococcus aureus

HA-MRSA

hospital-acquired methicillin-resistant Staphylococcus aureus

CA-MRSA

community-acquired methicillin-resistant Staphylococcus aureus

SCCmec

staphylococcal cassette chromosome mec

VISA

vancomycin intermediate Staphylococcus aureus

VRSA

vancomycin resistance Staphylococcus aureus

MDR

multi-drug resistant

HCW

health care worker

ICU

intensive care unit

cCU

cardiac care unit

ENT

ear, nose, and throat

MIC

minimal inhibitory concentration

CLSI

clinical and laboratory standards institute

AST

Page 18/25 
Antibiotic susceptibility test

R

resistant

I

intermediate

S

susceptible.

agr

accessory gene regulator

$\mathbf{P}$

Penicillin

CF

Cefoxitin

VA

Vancomycin

GM

Gentamicin

E

Erythromycin

TE

Tetracycline

CIP

Ciprofloxacin

FD

Nitrofurantoin

CC

Clindamycin

C

Chloramphenicol

RIF

Rifampin

LNZ

Linezolid

SYN

Quinupristin-Dalfopristin

\section{Declarations}

\section{Ethics approval and consent to participate}

The study protocol was approved by the Ethics Committee of Golestan university of medical sciences (No. 31078693122419). The study protocol was in accordance with the ethical guidelines of the 1975 Declaration of Helsinki. For hospital personnel, written informed consent was obtained before sampling. 


\section{Consent for publication}

Not applicable.

\section{Availability of data and materials}

All data generated or analyzed during this study are included in this published article.

\section{Competing interests}

The authors declare that they have no conflict of interest.

\section{Acknowledgements}

This research was supported by Golestan University of Medical Sciences, Gorgan, Iran (No. 931219278).

\section{References}

1. Grema HA, Geidam YA, Gadzama GB, Ameh JA, Suleiman A. Methicillin resistant Staphylococcus aureus (MRSA): a review. Adv Anim Vet Sci. 2015;3(2):79-98.

2. Goudarzi M, Goudarzi H, Figueiredo AMS, Udo EE, Fazeli M, Asadzadeh M, et al. Molecular characterization of methicillin resistant Staphylococcus aureus strains isolated from intensive care units in Iran: ST22SCCmec IV/t790 emerges as the major clone. PLoS One. 2016;11(5):e0155529.

3. Wang X, Li X, Liu W, Huang W, Fu Q, Li M. Molecular characteristic and virulence gene profiles of communityassociated methicillin-resistant Staphylococcus aureus isolates from pediatric patients in Shanghai, China. Front Microbiol. 2016;7:1818.

4. Qiao Y, Dong F, Song W, Wang L, Yang Y, Shen X. Hospital-and community-associated methicillin-resistant Staphylococcus aureus: a 6-year surveillance study of invasive infections in Chinese children. Acta Paediatr. 2013;102(11):1081-6.

5. Xiao Y, Wei Z, Shen P, Ji J, Sun Z, Yu H, et al. Bacterial-resistance among outpatients of county hospitals in China: significant geographic distinctions and minor differences between central cities. Microbes Infect. 2015;17(6):417-25.

6. Hiramatsu K, Katayama Y, Yuzawa H, Ito T. Molecular genetics of methicillin-resistant Staphylococcus aureus. Int J Med Microbiol. 2002;292(2):67-74.

7. Gastmeier P, Schwab F, Meyer E, Geffers C. Excess mortality and prolongation of stay due to bloodstream infections caused by multiresistant pathogens in Germany. Dtsch Med Wochenschr. 2012;137(3435):1689-92.

8. Fasihi Y, Kiaei S, Kalantar-Neyestanaki D. Characterization of SCCmec and spa types of methicillin-resistant Staphylococcus aureus isolates from health-care and community-acquired infections in Kerman, Iran. J Epidemiol Glob Health. 2017;7(4):263-7. 
9. Vitale M, Gaglio S, Galluzzo P, Cascone G, Piraino C, Di Marco Lo Presti V, et al. Antibiotic resistance profiling, analysis of virulence aspects and molecular genotyping of Staphylococcus aureus isolated in Sicily, Italy. Foodborne Pathog Dis. 2018;15(3):177-85.

10. Cheraghi S, Pourgholi L, Shafaati M, Fesharaki SH, Jalali A, Nosrati R, et al. Analysis of virulence genes and accessory gene regulator (agr) types among methicillin-resistant Staphylococcus aureus strains in Iran. J Glob Antimicrob Resist. 2017;10:315-20.

11. Xie X, Dai X, Ni L, Chen B, Luo Z, Yao Y, et al. Molecular epidemiology and virulence characteristics of Staphylococcus aureus nasal colonization in medical laboratory staff: comparison between microbiological and non-microbiological laboratories. BMC Infect Dis. 2018;18(1):122.

12. Wertheim HF, Melles DC, Vos MC, van Leeuwen W, van Belkum A, Verbrugh HA, et al. The role of nasal carriage in Staphylococcus aureus infections. Lancet Infect Dis. 2005;5(12):751-62.

13. Feil EJ, Cooper JE, Grundmann H, Robinson DA, Enright MC, Berendt T, et al. How clonal is Staphylococcus aureus? J Bacteriol. 2003;185(11):3307-16.

14. Goudarzi M, Seyedjavadi SS, Nasiri MJ, Goudarzi H, Nia RS, Dabiri H. Molecular characteristics of methicillinresistant Staphylococcus aureus (MRSA) strains isolated from patients with bacteremia based on MLST, SCCmec, spa, and agr locus types analysis. Microb Pathog. 2017;104:328-35.

15. Liang B, Mai J, Liu Y, Huang Y, Zhong H, Xie Y, et al. Prevalence and Characterization of Staphylococcus aureus Isolated From Women and Children in Guangzhou, China. Front Microbiol. 2018;9:2790.

16. Zhang W, Shen X, Zhang H, Wang C, Deng Q, Liu L, et al. Molecular epidemiological analysis of methicillinresistant Staphylococcus aureus isolates from Chinese pediatric patients. Eur J Clin Microbiol Infect Dis. 2009;28(7):861-4.

17. Cha HY, Moon DC, Choi CH, Oh JY, Jeong YS, Lee YC, et al. Prevalence of the ST239 clone of methicillinresistant Staphylococcus aureus and differences in antimicrobial susceptibilities of ST239 and ST5 clones identified in a Korean hospital. J Clin Microbiol. 2005;43(8):3610-4.

18. Ledda A, Price JR, Cole K, Llewelyn MJ, Kearns AM, Crook DW, et al. Re-emergence of methicillin susceptibility in a resistant lineage of Staphylococcus aureus. J Antimicrob Chemother. 2017;72(5):1285-8.

19. Rahimi S, Amini A, Cheraghali F, Tabbaraei A, Ghaemi EA. The frequency of MRSA carriers in health care workers in Gorgan, North of Iran. HealthMED. 2011;5(6 SUPP):1885-90.

20. Azimian A, Havaei SA, Fazeli H, Naderi M, Ghazvini K, Samiee SM, et al. Genetic characterization of a vancomycin-resistant Staphylococcus aureus isolate from the respiratory tract of a patient in a university hospital in northeastern Iran. J Clin Microbiol. 2012;50(11):3581-5.

21. Weinstein MP, Limbago B, Patel J, Mathers A, Campeau S, Mazzulli T, et al. Performance standards for antimicrobial disk susceptibility tests. M100 standard. 28th ed. Wayne: Clinical and Laboratory Standards Institute; 2018.

22. Clark N, Cooksey R, Hill B, Swenson J, Tenover F. Characterization of glycopeptide-resistant enterococci from US hospitals. Antimicrob Agents Chemother. 1993;37(11):2311-7.

23. Kehrenberg C, Schwarz S. Distribution of florfenicol resistance genes fexA and cfr among chloramphenicolresistant Staphylococcus isolates. Antimicrob Agents Chemother. 2006;50(4):1156-63.

24. Lin J, Zhang T, Bai C, Liang J, Ye J, Yao Z. School environmental contamination of methicillin-sensitive Staphylococcus aureus as an independent risk factor for nasal colonization in schoolchildren: An 
observational, cross-sectional study. PLoS One. 2018;13(11):e0208183.

25. Gostev V, Kruglov A, Kalinogorskaya O, Dmitrenko O, Khokhlova O, Yamamoto T, et al. Molecular epidemiology and antibiotic resistance of methicillin-resistant Staphylococcus aureus circulating in the Russian Federation. Infect Genet Evol. 2017;53:189-94.

26. Cirkovic I, Trajkovic J, Hauschild T, Andersen PS, Shittu A, Larsen AR. Nasal and pharyngeal carriage of methicillin-resistant Staphylococcus sciuri among hospitalised patients and healthcare workers in a Serbian university hospital. PLoS One. 2017;12(9):e0185181.

27. Boye K, Bartels MD, Andersen IS, Moeller JA, Westh H. A new multiplex PCR for easy screening of methicillinresistant Staphylococcus aureus SCCmec types I-V. Clin Microbiol Infect. 2007;13(7):725-7.

28. Harmsen D, Claus H, Witte W, Rothgänger J, Claus H, Turnwald D, et al. Typing of methicillin-resistant Staphylococcus aureus in a university hospital setting by using novel software for spa repeat determination and database management. J Clin Microbiol. 2003;41(12):5442-8.

29. Shopsin B, Mathema B, Alcabes P, Said-Salim B, Lina G, Matsuka A, et al. Prevalence of agr specificity groups among Staphylococcus aureus strains colonizing children and their guardians. J Clin Microbiol. 2003;41(1):456-9.

30. Enright MC, Day NP, Davies CE, Peacock SJ, Spratt BG. Multilocus sequence typing for characterization of methicillin-resistant and methicillin-susceptible clones ofStaphylococcus aureus. J Clin Microbiol. 2000;38(3):1008-15.

31. Conceicao T, Aires-de-Sousa M, Füzi M, Toth A, Paszti J, Ungvári E, et al. Replacement of methicillin-resistant Staphylococcus aureus clones in Hungary over time: a 10-year surveillance study. Clin Microbiol Infect. 2007;13(10):971-9.

32. Ellington MJ, Ganner M, Warner M, Cookson BD, Kearns AM. Polyclonal multiply antibiotic-resistant methicillin-resistant Staphylococcus aureus with Panton-Valentine leucocidin in England. J Antimicrob Chemother. 2010;65(1):46-50.

33. Namvar AE, Afshar M, Asghari B, Lari AR. Characterisation of SCCmec elements in methicillin-resistant Staphylococcus aureus isolated from burn patients. Burns. 2014;40(4):708-12.

34. Emaneini M, Bigverdi R, Kalantar D, Soroush S, Jabalameli F, Khoshgnab BN, et al. Distribution of genes encoding tetracycline resistance and aminoglycoside modifying enzymes in Staphylococcus aureus strains isolated from a burn center. Ann Burns Fire Disasters. 2013;26(2):76-80.

35. Darban-Sarokhalil D, Khoramrooz SS, Marashifard M, Hosseini SAAM, Parhizgari N, Yazdanpanah M, et al. Molecular characterization of Staphylococcus aureus isolates from southwest of Iran using spa and SCCmec typing methods. Microb Pathog. 2016;98:88-92.

36. Bartoloni A, Riccobono E, Magnelli D, Villagran AL, Di Maggio T, Mantella A, et al. Methicillin-resistant Staphylococcus aureus in hospitalized patients from the Bolivian Chaco. Int J Infect Dis. 2015;30:156-60.

37. O’Malley S, Emele F, Nwaokorie F, Idika N, Umeizudike A, Emeka-Nwabunnia I, et al. Molecular typing of antibiotic-resistant Staphylococcus aureus in Nigeria. J Infect Public Health. 2015;8(2):187-93.

38. Wang W-Y, Chiueh T-S, Sun J-R, Tsao S-M, Lu J-J. Molecular typing and phenotype characterization of methicillin-resistant Staphylococcus aureus isolates from blood in Taiwan. PLoS One. 2012;7(1):e30394.

39. Awadalla H, Khalil I, Bassim H, Ahmed M, Wahba L. Molecular typing of methicilin-resistant Staphylococcus aureus isolates at Ain Shams University Hospital, Egypt. Afr J Microbiol Res. 2010;4(15):1639-46.

Page 22/25 
40. Abbasian S, Farahani NN, Mir Z, Alinejad F, Haeili M, Dahmardehei M, et al. Genotypic characterization of Staphylococcus aureus isolated from a burn centre by using agr, spa and SCCmec typing methods. New Microbe New Infect. 2018;26:15-9.

41. Song W, Lee KM, Kang HJ, Shin DH, Kim DK. Microbiologic aspects of predominant bacteria isolated from the burn patients in Korea. Burns. 2001;27(2):136-9.

42. Shore AC, Rossney AS, O'Connell B, Herra CM, Sullivan DJ, Humphreys H, et al. Detection of SCCmecassociated DNA Segments in multiresistant methicillin-susceptible Staphylococcus aureus (MSSA) and identification of Staphylococcus epidermidis ccrAB4 in both methicillin-resistant S. aureus (MRSA) and MSSA. Antimicrob Agents Chemother. 2008;52(12):4407-19.

43. Vaez H, Tabaraei A, Moradi A, Ghaemi EA. Evaluation of methicillin resistance Staphylococcus aureus isolated from patients in Golestan province-north of Iran. Afr J Microbiol Res. 2011;5(4):432-6.

44. Khosravi AD, Hoveizavi H, Farshadzadeh Z. The prevalence of genes encoding leukocidins in Staphylococcus aureus strains resistant and sensitive to methicillin isolated from burn patients in Taleghani Hospital, Ahvaz, Iran. Burns. 2012;38(2):247-51.

45. Heidari M, Momtaz H, Madani M. Detection of the antibiotic resistance genes in Staphylococcus aureus isolated from human infections and bovine mastitis. Afr J Microbiol Res. 2011;5(28):5132-6.

46. Sepehriseresht S, Boroumand MA, Pourgholi L, Anvari MS, Habibi E, Tabrizi MS, et al. Emergence of mupirocin-resistant MRSA among Iranian clinical isolates. Comp Clin Path. 2013;22(4):717-21.

47. Havaei SA, Ghanbari F, Rastegari AA, Azimian A, Khademi F, Hosseini N, et al. Molecular typing of hospitalacquired Staphylococcus aureus isolated from Isfahan, Iran. Int Sch Res Not. 2014;2014:1-6.

48. Hasani A, Sheikhalizadeh V, Hasani A, Naghili B, Valizadeh V, Nikoonijad AR. Methicillin resistant and susceptible Staphylococcus aureus: Appraising therapeutic approaches in the Northwest of Iran. Iran J Microbiol. 2013;5(1):56-62.

49. Fatholahzadeh B, Emaneini M, Gilbert G, Udo E, Aligholi M, Modarressi MH, et al. Staphylococcal cassette chromosome mec (SCC mec) analysis and antimicrobial susceptibility patterns of methicillin-resistant Staphylococcus aureus (MRSA) isolates in Tehran, Iran. Microb Drug Resist. 2008;14(3):217-20.

50. Japoni A, Jamalidoust M, Farshad S, Ziyaeyan M, Alborzi A, Japoni S, et al. Characterization of SCCmec types and antibacterial susceptibility patterns of methicillin-resistant Staphylococcus aureus in Southern Iran. Jpn J Infect Dis. 2011;64(1):28-33.

51. Parhizgari N, Khoramrooz SS, Hosseini M, Asghar SA, Marashifard M, Yazdanpanah M, et al. High frequency of multidrug-resistant Staphylococcus aureus with SCCmec type III and Spa types t037 and t631 isolated from burn patients in southwest of Iran. APMIS. 2016;124(3):221-8.

52. Zetola N, Francis JS, Nuermberger EL, Bishai WR. Community-acquired meticillin-resistant Staphylococcus aureus: an emerging threat. Lancet Infect Dis. 2005;5(5):275-86.

53. Ghasemian A, Peerayeh SN, Bakhshi B, Mirzaee M. Detection of accessory gene regulator groups genes and cassette chromosome mec types among Staphylococcus aureus isolated from intensive care unit patients. Asian Pac J Trop Dis. 2015;5(2):153-7.

54. Nowrouzian FL, Dauwalder O, Meugnier H, Bes M, Etienne J, Vandenesch F, et al. Adhesin and superantigen genes and the capacity of Staphylococcus aureus to colonize the infantile gut. J Infect Dis.

2011;204(5):714-21. 
55. Asadollahi P, Farahani NN, Mirzaii M, Khoramrooz SS, Van Belkum A, Asadollahi K, et al. Distribution of the most prevalent spa types among clinical isolates of methicillin-resistant and-susceptible Staphylococcus aureus around the world: A review. Front Microbiol. 2018;9:163.

56. Emaneini M, Khoramrooz SS, Taherikalani M, Jabalameli F, Aligholi M. Molecular characterization of Staphylococcus aureus isolated from children with adenoid hypertrophy: emergence of new spa types t7685 and t7692. Int J Pediatr Otorhinolaryngol. 2011;75(11):1446-9.

57. Kong H, Yu F, Zhang W, Li X, Wang H. Molecular epidemiology and antibiotic resistance profiles of methicillinresistant Staphylococcus aureus strains in a tertiary hospital in China. Front Microbiol. 2017;8:838.

58. Chen H, Liu Y, Jiang X, Chen M, Wang H. Rapid change of methicillin-resistant Staphylococcus aureus clones in a Chinese tertiary care hospital over a 15-year period. Antimicrob Agents Chemother. 2010;54(5):1842-7.

59. Shahsavan S, Jabalameli L, Maleknejad P, Aligholi M, Imaneini H, Jabalameli F, et al. Molecular analysis and antimicrobial susceptibility of methicillin resistant Staphylococcus aureus in one of the hospitals of Tehran University of Medical Sciences: high prevalence of sequence type 239 (ST239) clone. Acta Microbiol Immunol Hung. 2011;58(1):31-9.

60. Havaei SA, Vidovic S, Tahmineh N, Mohammad K, Mohsen K, Starnino S, et al. Epidemic methicillinsusceptible Staphylococcus aureus lineages are the main cause of infections at an Iranian university hospital. J Clin Microbiol. 2011;49(11):3990-3.

61. Udo EE, Al-Lawati B-H, Al-Muharmi Z, Thukral S. Genotyping of methicillin-resistant Staphylococcus aureus in the Sultan Qaboos University Hospital, Oman reveals the dominance of Panton-Valentine leucocidinnegative ST6-IV/t304 clone. New Microbes New Infect. 2014;2(4):100-5.

62. Ko KS, Lee J-Y, Suh JY, Oh WS, Peck KR, Lee NY, et al. Distribution of major genotypes among methicillinresistant Staphylococcus aureus clones in Asian countries. J Clin Microbiol. 2005;43(1):421-6.

63. El-Mahdy T, El-Ahmady M, Goering RV. Molecular characterization of methicillin-resistant Staphylococcus aureus isolated over a 2-year period in a Qatari hospital from multinational patients. Clin Microbiol Infect. 2014;20(2):169-73.

64. Shekarabi M, Hajikhani B, Chirani AS, Fazeli M, Goudarzi M. Molecular characterization of vancomycinresistant Staphylococcus aureus strains isolated from clinical samples: A three year study in Tehran, Iran. PLoS One. 2017;12(8):e0183607.

65. Bozdogan B, Ednie L, Credito K, Kosowska K, Appelbaum PC. Derivatives of a vancomycin-resistant Staphylococcus aureus strain isolated at Hershey Medical Center. Antimicrob Agents Chemother. 2004;48(12):4762-5.

\section{Figures}




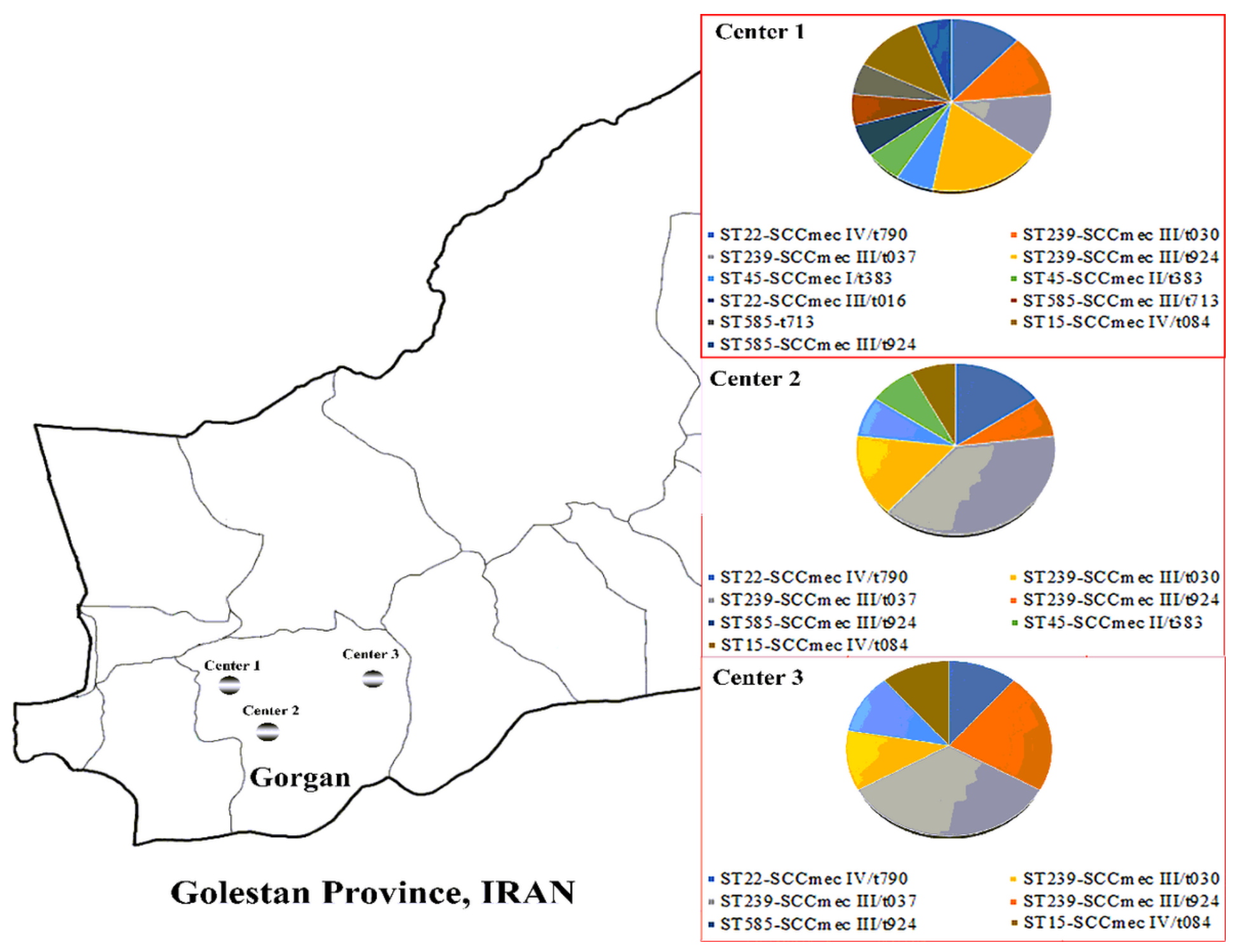

Figure 1

Distribution of MRSA types at the three clinical centers in Gorgan, Iran. Abbreviations: MRSA, methicillin-resistant Staphylococcus aureus. 